Ion cyclotron resonance heating with consistent finite orbit widths and anisotropic equilibria

This article has been downloaded from IOPscience. Please scroll down to see the full text article.

2011 Plasma Phys. Control. Fusion 53054010

(http://iopscience.iop.org/0741-3335/53/5/054010)

View the table of contents for this issue, or go to the journal homepage for more

Download details:

IP Address: 128.178.125.164

The article was downloaded on 13/04/2011 at 09:55

Please note that terms and conditions apply. 


\title{
Ion cyclotron resonance heating with consistent finite orbit widths and anisotropic equilibria
}

\author{
M Jucker ${ }^{1}$, J P Graves ${ }^{1}$, W A Cooper ${ }^{1}$ and T Johnson ${ }^{2}$ \\ ${ }^{1}$ Ecole Polytechnique Fédérale de Lausanne (EPFL), Centre de Recherches en Physique des \\ Plasmas, Association EURATOM-Confédération Suisse, CH-1015 Lausanne, Switzerland \\ ${ }^{2}$ Euratom-VR Association, EES, KTH, Stockholm, Sweden \\ E-mail: martin.jucker@a3.epfl.ch
}

Received 7 September 2010, in final form 7 December 2010

Published 7 April 2011

Online at stacks.iop.org/PPCF/53/054010

\begin{abstract}
Minority ion cyclotron resonance heating is studied using the self-consistent numerical model SCENIC. This model includes 3D geometries with full shaping and anisotropic pressure effects, warm contributions to the dielectric tensor and full orbit effects. It evolves the equilibrium, wave field and hot particle distribution function iteratively until a self-consistent solution is found. We will show applications to JET-like two-dimensional equilibria with minority heating scenarios. The effects due to different heating locations on the hot particle distribution function, the hot dielectric tensor and the equilibrium will be studied for symmetric wave injection. Finally, the RF-induced particle pinch is investigated using asymmetric wave injection.
\end{abstract}

(Some figures in this article are in colour only in the electronic version)

\section{Introduction}

The deployment of high-power radio frequency waves in the ion cyclotron range (ICRF) constitutes an important operational facility in many plasma devices, including ITER. ICRF is not only used for pure heating of the plasma, i.e. ion cyclotron resonance heating (ICRH), but also for the generation of non-inductive current through ion cyclotron current drive (ICCD). Applied to plasma species with low concentrations (minority scheme), highly localized heating effects can be generated, and ICCD can therefore be used to control MHD instabilities, e.g. sawteeth as in [1]. The physics involved span a region that encompasses MHD equilibrium, wave propagation and absorption, kinetic effects from wave-particle interactions and also wide orbit effects. As a result, observations include many mutual interactions and the effects of single ingredients are difficult to filter out from measurements. It is therefore important to develop numerical tools which include as many physical mechanisms as possible to simulate radio frequency (RF) scenarios accurately. For a self-consistent numerical description of these 
effects, the integrated model SCENIC [2], capable of iterated ICRF simulations, will be used. The MHD equilibrium is computed using VMEC [3], the wave fields and wave numbers by LEMan [4], and the evolution of the distribution function with VENUS [5]. These three codes are used iteratively in order to allow for a self-consistent solution. Similar models have been developed earlier, for instance SELFO [6] for circular cross sections, the package AORSACQL3D [7] assuming zero orbit widths or, very recently, AORSA-ORBIT-RF [8]. Finite orbit width effects become very important in the energy range of the non-Maxwellian tails developing in ICRF schemes, and neglecting them represents an important constraint to any numerical model. SELFO has been successfully applied to ICRF computations, but it cannot account for the effects of plasma shaping and upshift in the parallel wave number. The AORSA-ORBITRF package appears to be a promising new candidate for $2 \mathrm{D}$ self-consistent calculations, but it only includes changes in magnetic moment due to wave-particle interactions, which does not include RF-induced particle drifts [9, 10]. Also, only two iterations and simulations for about one slowing down time have been reported [8], which, as we will show, might not be enough for finding a converged solution. As explained earlier, ICRF is applied to a given plasma either for heating or driving currents, both of which will unavoidably have an effect on the equilibrium itself. First attempts to evolve the equilibrium have been undertaken in [11]. These authors studied lower hybrid current drive scenarios and allowed for a current density variation determined by a simplified model comprising a unidimensional Fokker-Planck code coupled to a ray tracing method. In addition to the RF-induced current, the arising pressure anisotropy can have a direct effect on plasma shaping [12]. This is why we developed with SCENIC a code package which is able to include the (anisotropic) equilibrium in the self-consistent computations, and this feature represents a complete novelty. Moreover, the package is entirely based on codes being able to deal with $3 \mathrm{D}$ geometries, such that we will, in the future, not be limited to studying two-dimensional cases. The numerical model will be described in section 2 . From the large variety of possible RF scenarios, we will concentrate on minority species heating in JET-like equilibria in section 3. Here, we will simulate a case of two injected RF waves with equal toroidal wave numbers, but opposite directions ( \pm 90 phasing), applied at different locations in the plasma. This scenario minimizes the generation of Fisch currents [13], and we will thus be able to study the effects of wide and non-standard orbits, including detrapping of resonant particles, in more detail. Then, we will show the emergence of a high-energy tail in the minority species distribution function and its consequences on the dielectric tensor, wave field and equilibrium.

\section{The code package SCENIC}

The code package SCENIC is described in detail in [2] and only a short overview will be given here. Figure 1 shows a flow diagram of SCENIC, with the input and output flows between the three codes VMEC, LEMan and VENUS. Self-consistent solutions for minority ICRH can be obtained by iterating between the three before mentioned codes until convergence is obtained. The final results encompass the full minority species distribution function and its integrated moments, a consistent magnetic equilibrium and wave field, including detailed information on the dielectric tensors of the background and the minority species, RF power deposition and slowing down. All codes, and hence SCENIC, are in principle capable of general threedimensional geometries. However, we will concentrate on JET-like two-dimensional scenarios. For the equilibrium and wave field calculations, a bi-Maxwellian distribution function is used for the hot minority, allowing for pressure anisotropy and stronger poloidal dependence of the 


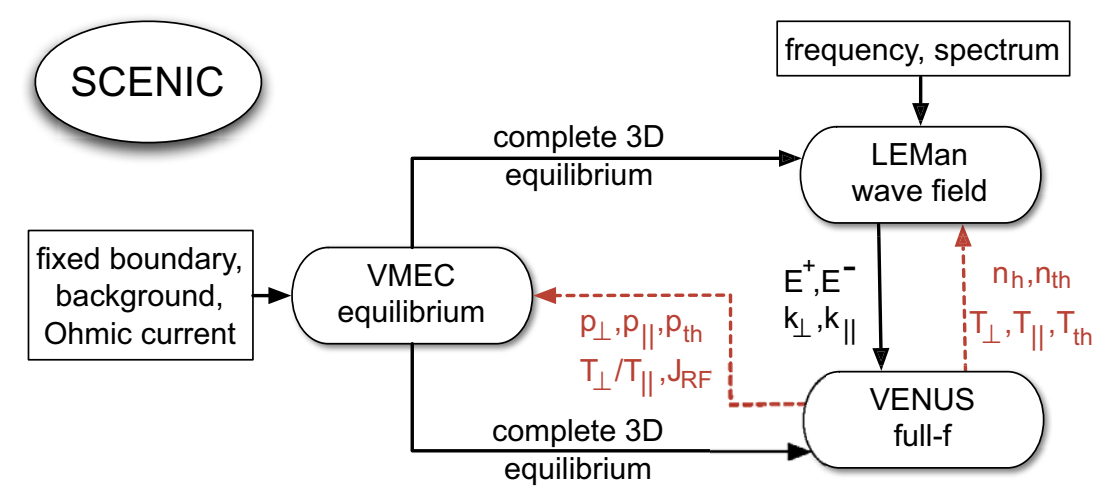

Figure 1. Flow diagram of the code package SCENIC. Rounded boxes denote the three coupled codes, rectangular boxes unchanging inputs, and additional text the flow of inputs and outputs. Solid arrows denote the flows for one iteration, dashed arrows the new inputs (VENUS outputs) needed for each new iteration. VENUS outputs refer to the minority species (hydrogen), with a hot anisotropic $(\perp, \|)$ and a thermal isotropic (subscript th) part. $n$ is the density, $T$ the temperature, $p$ the pressure and $J_{\mathrm{RF}}$ the total RF induced current. $E^{ \pm}$are the left- and right-hand polarized electric field components and $k$ is the wave vector.

pressure and dielectric tensor,

$$
F_{h}=\left(\frac{m}{2 \pi}\right)^{3 / 2} \frac{n_{\mathrm{c}}(\bar{s})}{T_{\perp}(\bar{s}) T_{\|}^{1 / 2}(\bar{s})} \exp \left[-\frac{\mu B_{\mathrm{c}}}{T_{\perp}(\bar{s})}-\frac{\left|E-\mu B_{\mathrm{c}}\right|}{T_{\|}(\bar{s})}\right],
$$

where $\bar{s}$ denotes the orbit-averaged radial position and is proportional to toroidal flux, $\mu$ is the magnetic moment, $n_{\mathrm{c}}(\bar{s})$ the density along the resonant layer $B=B_{\mathrm{c}}$ and $E$ the energy. In this form, the distribution function is written as a function of the constants of motion $(\bar{s}, \mu, E)$, and therefore satisfies $\boldsymbol{B} \cdot \nabla f$, corresponding to the lowest order expansion (in Larmor radius) of the Vlasov equation. $B_{\mathrm{c}}$ is a constant and denotes the location of the unshifted cyclotron resonance. Indeed, the wave frequency $\omega$ will be defined by this parameter through $\omega=Z_{i} e B_{\mathrm{c}} / m_{i}$. The inclusion of $B_{\mathrm{c}}$ also allows for the characteristic ear-shaped contours found for distribution functions in RF heating scenarios [14], as we will show in section 3.3. The equilibrium based on this distribution function is described in $[3,15]$. The anisotropic dielectric tensor and the computation of the parallel and perpendicular wave numbers are described in [2]. For a better understanding of this work, we repeat the explicit form of the anisotropic dielectric tensor implemented in LEMan for the hot minority in the appendix. After every VENUS run, the moments of the distribution function are fitted onto the bi-Maxwellian on which the equilibrium and wave field computations are based. With this, the latter two can be updated, thus completing the iteration [2]. However, it is important to note that the VENUS marker distribution is not subject to this fitting, and is allowed to evolve without any constraints throughout the simulation.

\section{High power scenarios}

In this work, we will use a JET-like D-shaped equilibrium with a magnetic field $B_{0}=2.94 \mathrm{~T}$, major radius $R_{0}=3 \mathrm{~m}$ and minor radius $a=1.17 \mathrm{~m}$. The central electron temperature and density are $3.5 \mathrm{keV}$ and $3.4 \times 10^{19} \mathrm{~m}^{-3}$, and we consider a deuterium plasma $\left(T_{\mathrm{D}}=T_{\mathrm{e}}\right)$ with a $3 \%$ hydrogen minority, having the exact same initial temperature and normalized density profiles as the background deuterium. This results in a volume-averaged beta of 


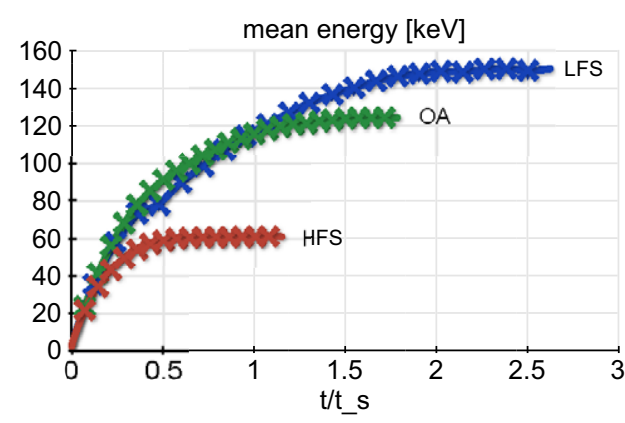

(a) Mean energy evolution. Crosses denote beginning of new iteration, where the equilibrium and the wave field are updated.

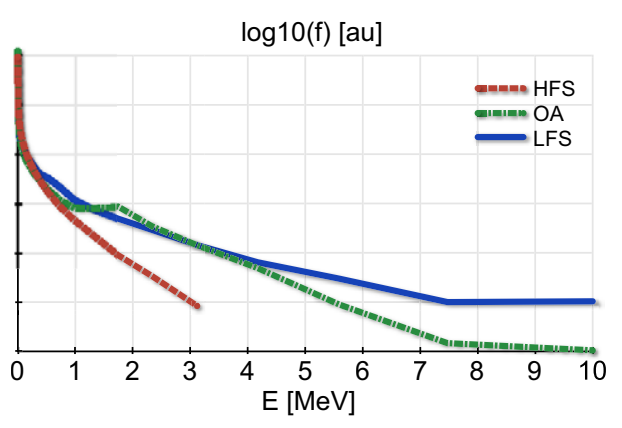

(b) Energy distribution function. The more the resonance moves towards the LFS, the more energetic are the tails.

Figure 2. Left: temporal evolution of the mean energy, equation (2). To the right, the final energy distribution functions with tails in the MeV range (integrated over real space). HFS stands for the resonance on the high-field side of the magnetic axis, OA for on axis heating and LFS for the resonance on the low-field side of the magnetic axis. Note the semilogarithmic scale.

$\left\langle\mu_{0} P / 2 B^{2}\right\rangle=0.13 \%$. Throughout this paper, the term background will be applied to the thermal Maxwellian deuterium ions and electrons. A part of the minority is not resonant with the wave and remains thermal and isotropic, and at the end of every VENUS run, the hydrogen minority is thus automatically split into two parts. The thermal part is treated Maxwellian as the background. The second part of the hydrogen minority represents the resonant tail, and will be referred to as hot minority, since this is the species treated bi-Maxwellian in the equilibrium and the wave field [2]. All simulations in this section are run using two waves, one co- and one counter-passing, in order to avoid Fisch currents [13], such that the somewhat more interesting finite orbit width effects can be elucidated. The toroidal wave numbers were $n_{\phi}= \pm 17$, the frequencies $52.6 \mathrm{MHz}$ (HFS), $48.5 \mathrm{MHz}(\mathrm{OA})$ and $38.7 \mathrm{MHz}$ (LFS), with a total deposited power of $12 \mathrm{MW}$. The simulations were performed with 96 (HFS) or 48 (OA,LFS) radial grid points and 30 poloidal Fourier modes in the equilibrium, 150 radial and 450 poloidal grid points (interpolated equilibrium) and 89 poloidal modes for the wave field computations, and four million markers in the guiding centre calculations.

\subsection{Moments of the distribution function}

During the advancing of the distribution using VENUS, we can use the mean energy per particle,

$$
\langle E\rangle \equiv \frac{1}{N} \int_{V} \frac{1}{2} m v^{2} f \mathrm{~d} V,
$$

with $N=\int_{V} f \mathrm{~d} V$ and $V$ the phase space volume, as a basic diagnostic of the state of the complete simulation. Figure 2(a) shows the evolution of the mean energy per particle of the minority species for the three studied scenarios, where each cross in the plot denotes one iteration, i.e. updating the equilibrium and the wave field with the information from VENUS. For the high-field side (HFS) case, we ran $16 \times 15 \mathrm{~ms}$ simulations, for the on axis (OA) case, $25 \times 15 \mathrm{~ms}$ simulations were used, and the low-field side (LFS) heating needed $22 \times 25 \mathrm{~ms}$ iterations. In the plots in figure $2(a)$, the time on the $x$-axis has been normalized to the electron slowing down time (OA) of $210 \mathrm{~ms}$. Only the HFS case reaches convergence within one slowing down time, whereas the other cases, especially the LFS case, are still far from the 


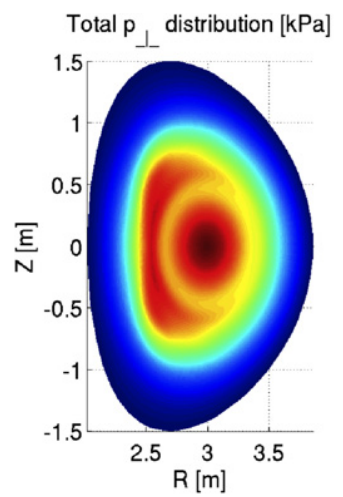

(a) HFS Iteration 16 .

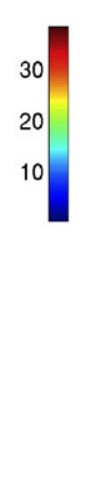

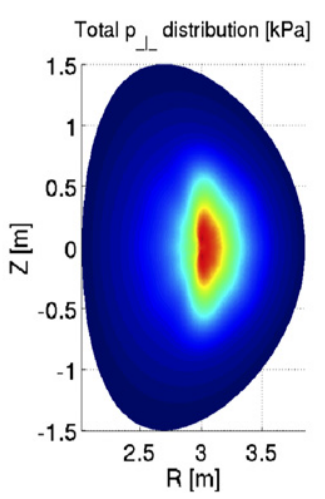

(b) OA Iteration 25

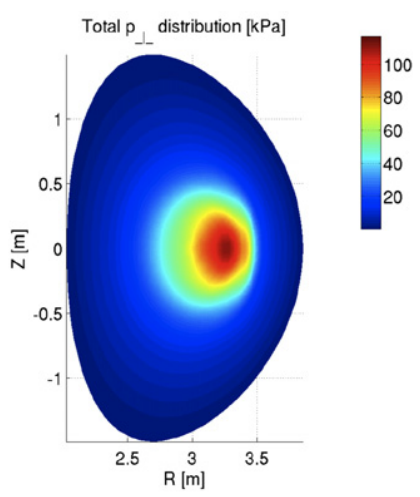

(c) LFS Iteration 20 .

Figure 3. Total perpendicular pressure plots for HFS, OA and LFS heating. Here, the pressure is not a flux surface quantity due to anisotropy.

steady state, and it is for the latter case imperative to iterate the codes further until at least 2.5 slowing down times. Note that this total duration of the simulation is at least an order of magnitude shorter than the resistive diffusion timescale, such that we can neglect the effects of current penetration due to resistivity in the equilibrium calculations. We would also like to note that this mean energy includes also parts of the hydrogen minority which do not interact with the RF field and remain thermal, and thus this quantity is not appropriate for determining the tail temperatures. For instance, the effective temperatures in the tail of the LFS case are of the order of $2 \mathrm{MeV}$, which is about ten times higher than the mean energy. Figure $2(b)$ shows the high-energy tails which are formed due to the wave-particle interactions. In all cases, we find effective temperatures in the $\mathrm{MeV}$ range, and 30-50\% of the hydrogen minority have an energy of more than ten times the background thermal temperature. The tails for OA and LFS heating are more energetic than the tail of the HFS resonance. The OA case has a distinct feature around $1.5 \mathrm{MeV}$, which is due to a secondary tail forming for the highest energies. This localized inversion of the gradient of the distribution function indicates a possibly unstable distribution.

In terms of heating the minority species, LFS and OA heating is more efficient than HFS heating, since the mean energy (and thus the total energy content) of the hydrogen is higher at the end of the simulation (about twice as high for LFS compared with OA). However, the simulation converges when the absorbed RF power equals the slowing down power, and the RF power is kept constant at $12 \mathrm{MW}$. Then, the lower energy content for the HFS heating can be explained by the fact that the resonant particles are mainly at the trapped-passing boundary, completing almost one full poloidal rotation before interacting again. Also, the orbits are wider for barely trapped particles, and so the resonant particles spend more time out of resonance and thus being slowed down by the background than the resonant particles of the OA heating case. Therefore, more power is going to the background, and the steady state is achieved at lower energies.

In figure 3 we show the final total perpendicular pressure for the three studied cases. It builds up rather quickly, forming an elongated structure along the resonant layer for the HFS case which becomes more localized as we move the resonance towards the LFS, where a very localized maximum appears. The other main difference between the LFS and the other two cases is that for the LFS, the maximum pressure is not so much located at the resonance at $R \approx 3.5 \mathrm{~m}$, but halfway between that location and the magnetic axis. In all three cases, the hot 


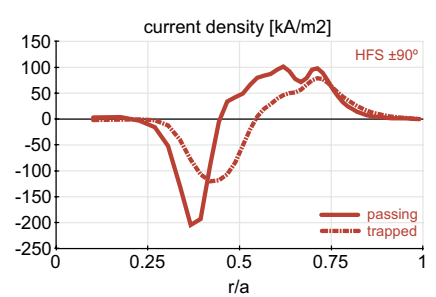

(a) HFS

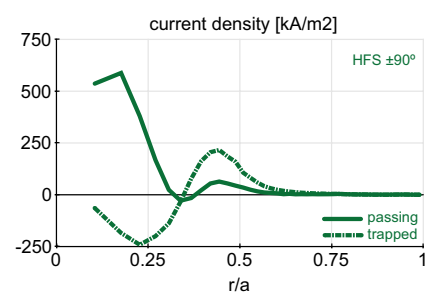

(b) OA

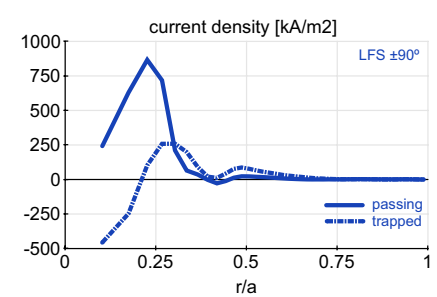

(c) LFS

Figure 4. RF-induced current density at the end of the simulation for HFS, OA and LFS heating.

pressure becomes locally more important than the background pressure, even if the thermal volume-averaged beta is always larger than its hot equivalent. We can note that the pressure cannot be considered a flux surface quantity anymore, which is known to have an effect on the Shafranov shift [12] as we will see later in this work.

As maybe the most interesting example of radial profiles, we turn to the RF-induced current through ICCD, figure 4. Note that we chose the RF spectrum to be toroidally symmetric, such that Fisch currents are negligible [13]. The currents shown here cannot be obtained using a zero orbit width Fokker-Planck code, since they have their origin in the finite orbit width effects [16] and preferential detrapping. For the HFS case, a simple dipolar structure is formed around the resonant layer at $r / a=0.5$. This dipole is due to finite orbit widths, and the fact that trapped particles always move in the counter-current direction on the inner and in the co-current direction on the outer leg of the orbit. The parallel current describes a dipole similar to the trapped current, as symmetric detrapping causes the parallel current to be similar to the trapped current. The current due to detrapping is dominant for the OA and LFS heating cases, figures $4(b)$ and $(c)$. Here, the passing current does not show the dipolar structure we have seen for HFS heating. The reason might be that non-standard orbits such as potato orbits are more abundant for these scenarios, and detrapping becomes asymmetric [17]. As a result, preferentially co-passing particles are produced [18]. Thus we see a large positive passing current forming, whereas the trapped particle current still assumes the dipolar structure we have seen before. A slight difference in the evolution of the current density for the LFS case is that the trapped particle current is dominant at first, since the resonance on the LFS produces deeply trapped particles which are not detrapped as easily as the orbits for the HFS heating. The final currents are the largest for LFS heating, which is in agreement with the highest energy content of figure $2(a)$.

\subsection{Power deposition}

The wave-particle resonance is satisfied if $\omega=n \Omega+k_{\|} v_{\|}$, where $\omega$ is the frequency of the wave, $n$ the harmonic (unity in our case), $\Omega$ the local cyclotron frequency, $k_{\|}$the parallel wave number and $v_{\|}$the resonant particle's parallel velocity. The Doppler shift $k_{\|} v_{\|}$is responsible for a broadening of the power deposition at high parallel temperatures. This is shown in figure 5, where the initially thin deposition along the resonant layer (not shown) is broadened by the RF heating, and especially for OA heating power is deposited almost up to the LFS plasma edge. The particles only interact with the wave field in the Doppler-broadened resonant layer, whereas they interact with the background ions and electrons through Coulomb collisions (slowing down) continuously, giving their energy to the background along their orbits. Due to the large orbit widths of the resonant particles one can therefore expect that the power going from the resonant ions to the background has a broader radial profile than the absorbed power 


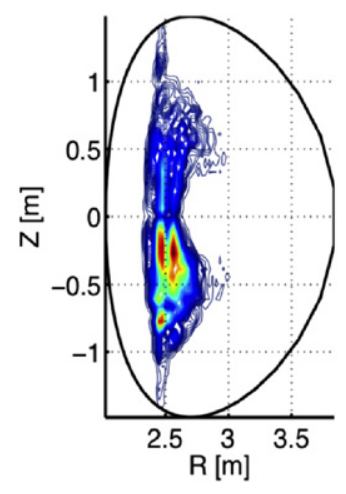

(a) HFS final power deposition.

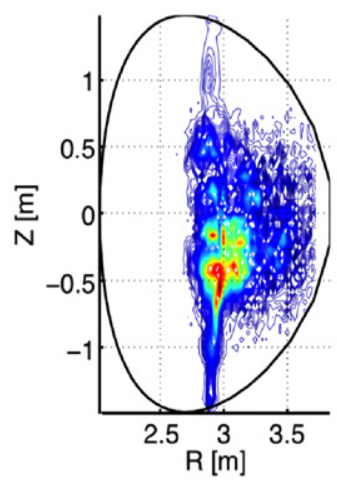

(b) OA final power deposition.

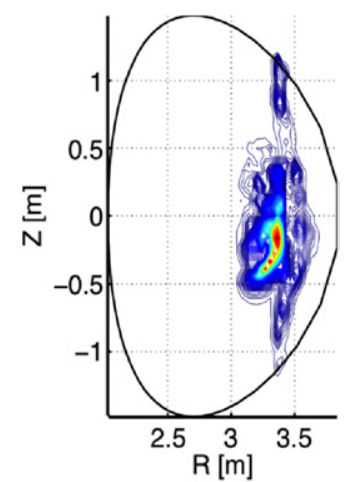

(c) LFS final power deposition.

Figure 5. Doppler broadening of the resonant layer. Shown is the deposition in the RF operators in VENUS at the end of the simulation.

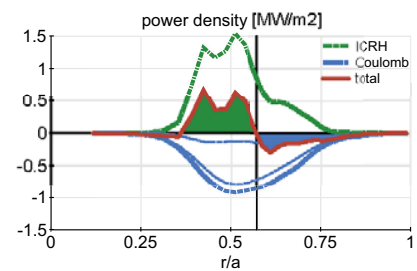

(a) HFS. Net heating between $\rho=0.35$ and $\rho=0.57$, net slowing down outside.

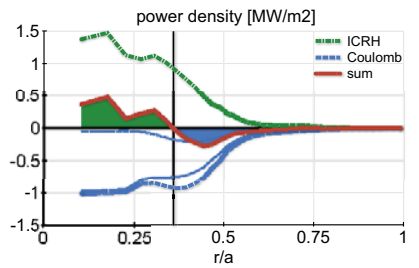

(b) OA. Net heating inside $\rho=$ 0.36 , net slowing down outside.

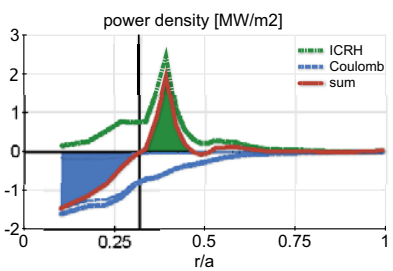

(c) LFS. Net heating between $\rho=0.32$ and $\rho=0.46$, net slowing down inside.

Figure 6. Radial profiles of power densities, showing regions of net heating $\left(P_{\mathrm{ICRH}}>P_{\mathrm{Coul}}\right.$, red shaded) and net slowing down $\left(P_{\text {ICRH }}<P_{\text {Coul }}\right.$, blue shaded $)$. Note that the integral of the resulting (red, thick) curve plus the small losses is zero in steady state. The thin dashed lines show the electron and ion contributions to the total slowing down.

from the wave. This is confirmed in figure 6 , where the power densities at a given flux surface are plotted. The thin blue and dashed lines represent the power going to the background ions (closer to the $x$-axis) and to the electrons (close to the total Coulomb curve). In the same figure, we see that in steady state, heating of the minority particles does not vanish, but that regions of net RF heating (local ICRH power absorption greater than slowing down, shaded in green) are balanced by regions of net slowing down (local ICRH power absorption smaller than slowing down, shaded in blue), such that

$$
\int\left(P_{\mathrm{ICRH}}+P_{\text {Coul }}+P_{\text {losses }}\right) \mathrm{d}^{2} x=0 \text {. }
$$

Note that the region of net slowing down is broader for the HFS than for the other cases, since the resonant particles describe larger orbits, resulting in the lower energy content of the minority species of figure $2(a)$.

\subsection{Model bi-Maxwellian}

For the equilibrium and the dielectric tensor, the model bi-Maxwellian distribution function (1) is implemented, and it is therefore important to check how good a model this represents. 
A way to directly compare the distribution function produced in VENUS and the model is to plot $f\left(v_{\|}, v_{\perp}\right)$ contours at a given spatial position. We chose for all cases the radial position of the cold resonant layer, but on the LFS midplane, $\theta=0$. We chose this position for two main reasons: the characteristic ear-shaped contours appear at high anisotropy, but they cannot be seen directly at the resonance where $B=B_{\mathrm{c}}$ [3], so that we can expect ear-shaped contours for HFS and OA and oval contours for LFS heating. Second, we expect the anisotropy to be the largest at that position, since it represents the average radial position of the particles with the strongest wave-particle interactions, at least for HFS and LFS. For OA heating, we decided to show the contours at the same position, since there is no other special position except the magnetic axis, where we do not have any grid points for statistics. In order to compare the VENUS with the model distribution, we re-initialized all markers according to the fit of the VENUS distribution onto the bi-Maxwellian, and time-averaged the distribution without applying any interactions, such that the result is equivalent to using the orbit-averaged radial position $\bar{s}$ in equation (1). This is only done at the very end of the simulation and for the proposed comparison, and is not necessary within the iterative scheme. In figure 7 , the ear-shaped features for the HFS case can very accurately be reproduced, with the tips along the (dashed) trapped-passing boundary, suggesting high number of barely trapped/passing particles as expected. For the OA case, the ears are inside the trapped-passing boundary, meaning that a larger population describes trapped orbits away from the trapped-passing boundary. However the model cannot reproduce the large asymmetry from the VENUS distribution OA case. Here, there is not only a strong asymmetry inside the trap cone, but also a larger population along the trapped-passing boundary, which indicates that in VENUS a considerable amount of particles are being detrapped, explaining the difference from the model. We can conclude from figure 7 that the model distribution is indeed able to treat the pressure anisotropy (responsible for the ear-shaped contours) and the high-energy tail except where special orbits and velocity and detrapping into barely passing particles appear.

For the equilibrium, the integrated moments of the distribution function are fitted to the model, most importantly the pressure profiles. Figure 8 gives the comparison between the directly integrated second moments of the distribution function in VENUS and the parallel and perpendicular pressure profiles resulting from the model. The match is indeed very good, with a small difference in anisotropy $\left(\sim p_{\perp} / p_{\|}\right)$at the resonance for the HFS and OA cases. For the LFS, the perpendicular pressure is smaller on the axis in the model. This is to a large part due to the smoothing of the raw profiles from VENUS, preventing anisotropy from peaking too much at the resonance. The match of the density profiles (figure 9) is even better, especially for the HFS case. The hot particle density is read by LEMan for the dielectric tensor. The latter cannot be compared with a 'raw' version from VENUS as the pressure and density profiles, and we will therefore simply show the form as it appears in the computations of LEMan. The initial form of the dielectric tensor is similar in all cases, showing a rather thin structure all along the resonant layer. In figure 10 the Doppler broadening can be observed in that the regions of maxima and minima are rather large at the end of each simulation. Also, the structures assume a certain poloidal dependence, which is due to the pressure anisotropy. The reason the structures are not extending up to the plasma edge in the final state is that the resonant particle density vanishes faster after applying RF than in the initial state, thus decreasing the amplitude of the dielectric tensor. Even if the total energy content is the highest for the LFS case, the Doppler broadening is not that extreme, such that the maxima of the dielectric tensor are very localized around the midplane. The reason lies in the fact that most of the energy is stored in perpendicular motion, which does not contribute to the Doppler shift.

The bi-Maxwellian model includes the finite orbit width effects in that it is written in terms of the constants of motion $\mu, E$ and $\bar{s}$. As a reminder, the latter denotes the average radial orbit 


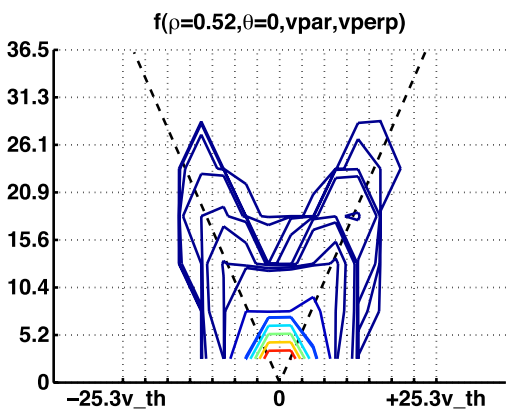

(a) HFS VENUS

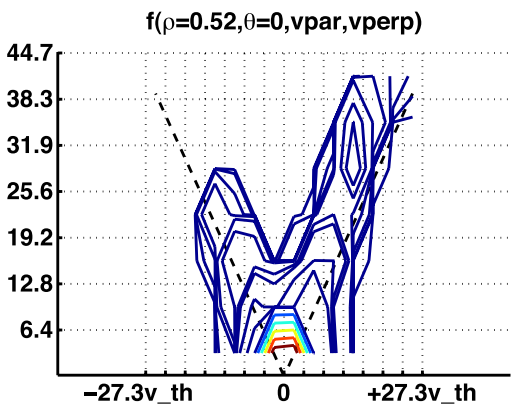

(c) OA VENUS

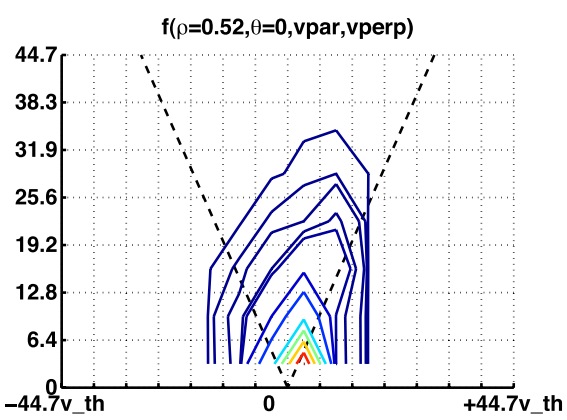

(e) LFS VENUS

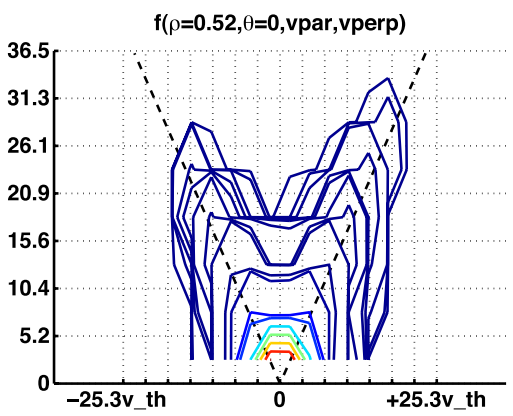

(b) HFS model

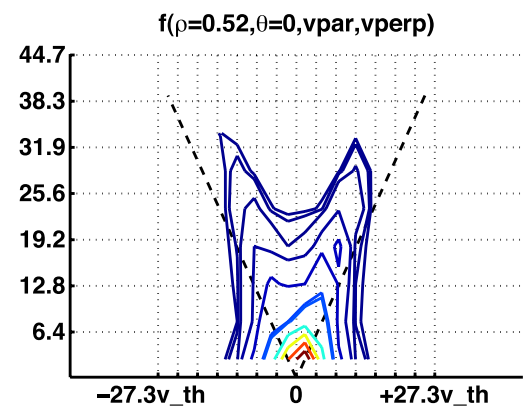

(d) OA model

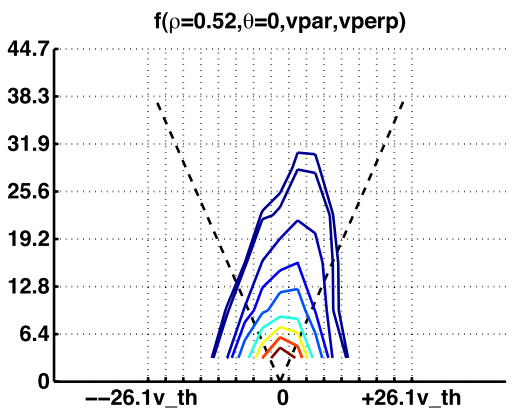

(f) LFS model

Figure 7. Comparison of the distribution function in VENUS with the bi-Maxwellian model. The match is very good where special orbits and detrapping do not play an important role.

position, which is, for an unperturbed orbit, a constant related to the toroidal angular momentum $P_{\phi}$. With these three constants an orbit of arbitrary width can be described, and therefore the model distribution function includes, e.g., a dipolar trapped particle current density. Although it is important to remember that the current density is fed into the equilibrium directly from VENUS and the model is not used here, it is still interesting to compare the RF induced current one can model with the analytical description of equation (1). This is a reason why we chose a symmetric wave spectrum, since the analytical model does not include the Fisch currents of zero width orbits which would arise for an asymmetric wave spectrum. The current from the VENUS simulations does not include the reaction of the background ions and electrons, and has to be diminished for the equilibrium computations. This is done using momentum conservation and quasi-neutrality and the balance of collision rates, given in [1] and references 


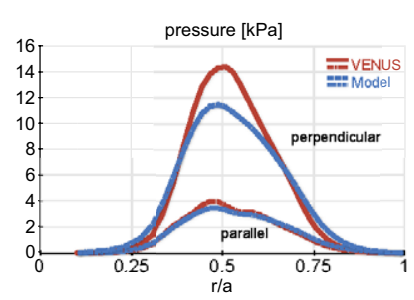

(a) HFS hot pressure profiles.

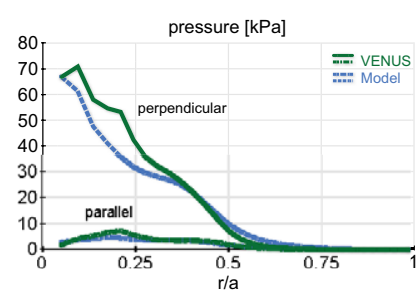

(b) OA hot pressure profiles.

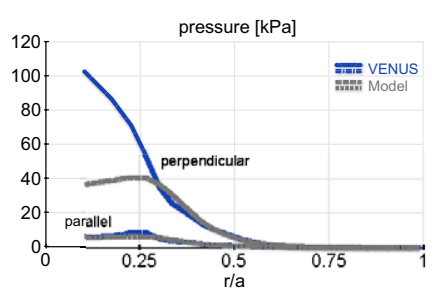

(c) LFS hot pressure profiles.

Figure 8. Comparison of the second moments from VENUS and the model distribution function. The curves labelled 'Model' have been obtained in the same way as in figure 7. The upper curve is hot perpendicular, the lower hot parallel pressure. These pressure profiles are read by VMEC for the equilibrium computations.

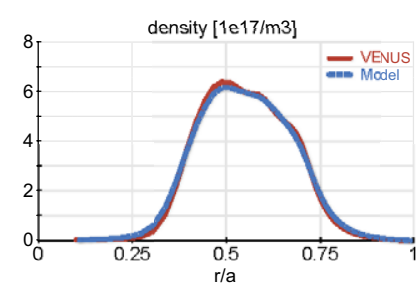

(a) HFS hot density profiles

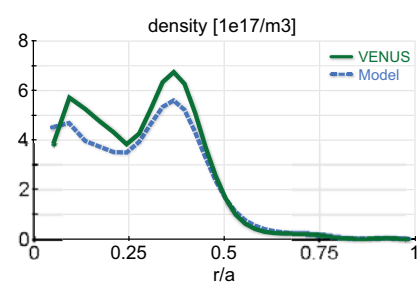

(b) OA hot density profiles.

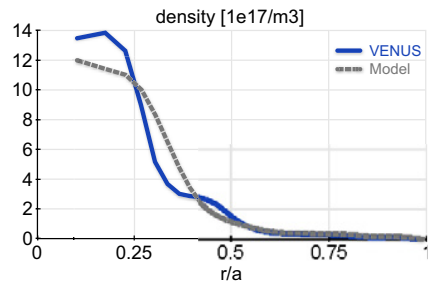

(c) LFS hot density profiles.

Figure 9. Comparison of the first moments from VENUS and the model distribution function. The curves labelled 'Model' have been obtained in the same way as in figure 7. The hot particle density from the model is used in LEMan for the dielectric tensor.

therein:

$$
\begin{aligned}
j_{\mathrm{VMEC}}^{\mathrm{RF}}=j_{\mathrm{VENUS}}^{\mathrm{RF}} & {\left[1-\frac{Z_{h}}{Z_{\mathrm{eff}}}-\frac{m_{h} \sum_{i} Z_{i} n_{i}\left(1-Z_{i} / Z_{\mathrm{eff}}\right)}{Z_{h} \sum_{i} n_{i} m_{i}}\right.} \\
+ & \left.1.46 \sqrt{\frac{r}{R_{0}}} A\left(Z_{\mathrm{eff}}\right)\left(\frac{Z_{h}}{Z_{\mathrm{eff}}}-\frac{m_{h} \sum_{i} n_{i} Z_{i}^{2}}{Z_{h} Z_{\text {eff }} \sum_{i} n_{i} m_{i}}\right)\right] .
\end{aligned}
$$

$A$ is a weak function of $Z_{\text {eff }}$ and is tabulated in [19]. For these simulations, we did not consider any impurities, such that $Z_{\text {eff }}=Z_{h}=1$ and $m_{i}=2 m_{h}$, and the drag current formula simplifies a lot to

$$
j_{\mathrm{VMEC}}^{\mathrm{RF}}=j_{\mathrm{VENUS}}^{\mathrm{RF}} 1.46 \sqrt{\frac{r}{R_{0}}} A(1) 0.5 .
$$

This means that the effective current in the equilibrium is underestimated with respect to a more realistic case with $Z_{\text {eff }}>1$, but this will be of more importance in the next section. Figure 11 compares the drag corrected current densities from VENUS (and thus the equilibrium computations) with the currents contained in the model. Solid and dashed-dotted lines denote the passing and trapped particle currents from VENUS, whereas dashed and lightly dashed lines show the passing and trapped particle currents contained in the model distribution. For the HFS heating case, the analytical model is indeed capable of modelling the trapped particle current, whereas it is somewhat further away from VENUS in the other cases. The reason for this is that for the HFS case, there are virtually no non-standard orbits, and thus the model contains all the information needed. However, as we have already seen in the contour plots of figure 7 , there is a strong asymmetry with respect to $v_{\|}$within the trap cone for the OA 


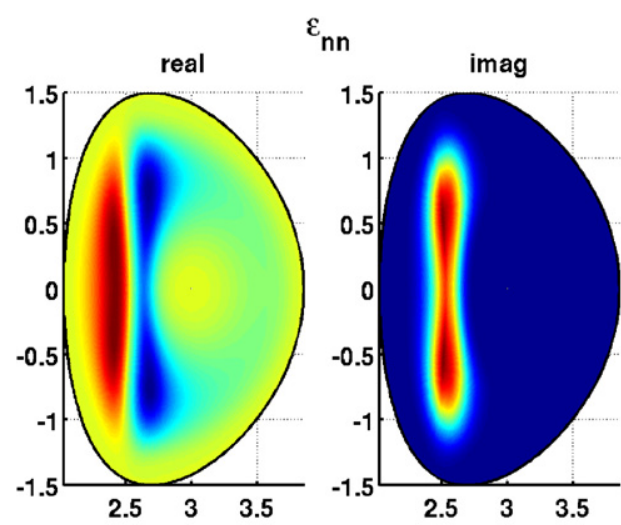

(a) HFS final.
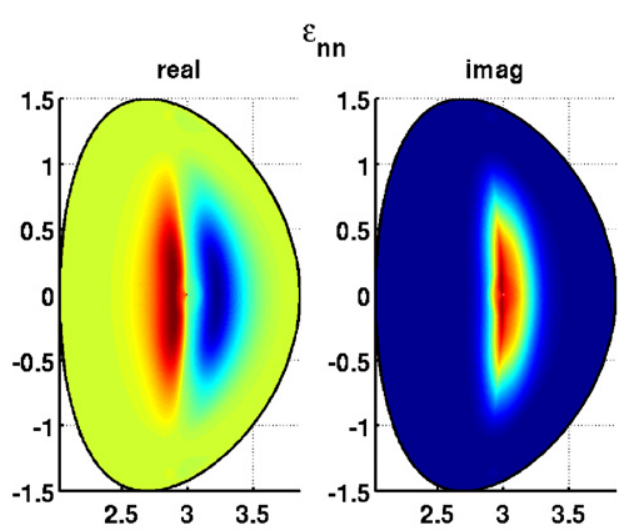

(b) OA final.
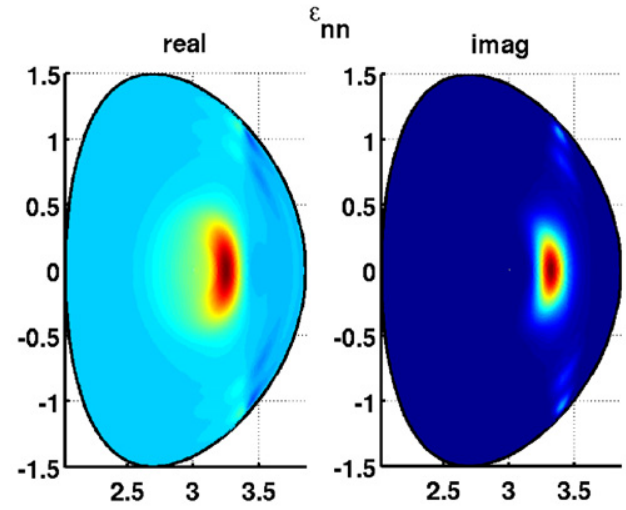

(c) LFS final.

Figure 10. Real and imaginary parts of the $\epsilon_{n n}$ elements defined in equations (A.1) and (A.4) in the converged state for each case. The maxima (minima) are considerably Doppler broadened, and assume a poloidal dependence due to high anisotropy.

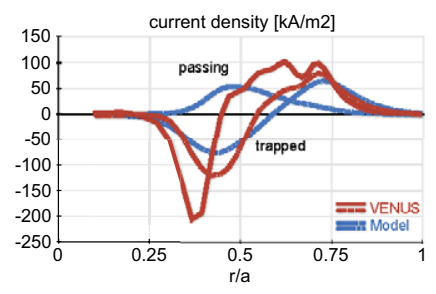

(a) HFS

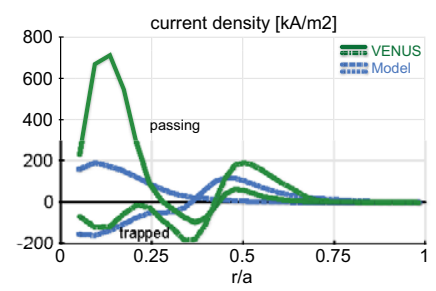

(b) OA

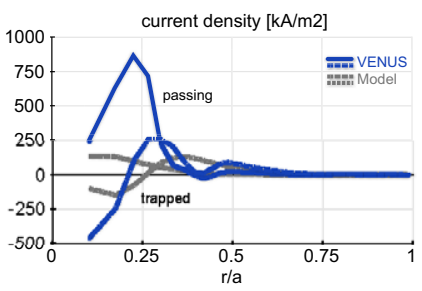

(c) LFS

Figure 11. Comparison of the real RF driven currents from VENUS (corrected by the drag current) with the currents coming from the analytical model distribution function. Trapped particle current can be modelled more accurately since its origin lies in finite orbit width effects. Solid (dasheddotted) lines denote passing (trapped) particle currents from VENUS, dashed (lightly dashed) lines passing (trapped) particle currents from the model bi-Maxwellian.

and LFS cases, which is missing in the model, and thus also the trapped current is somewhat different. The current density due to passing particles is mainly due to detrapping of resonant particles, a mechanism which is not included in the analytical model, since this would require a model that incorporates a different density of particles for co- and counter-passing ions [20]. 


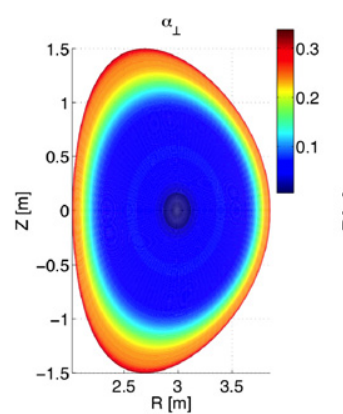

(a) Initial

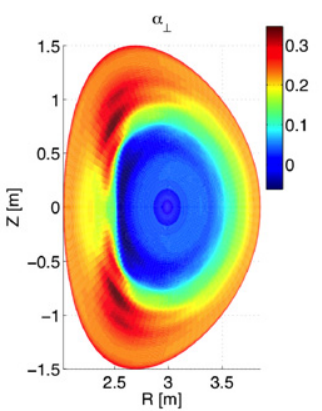

(b) HFS

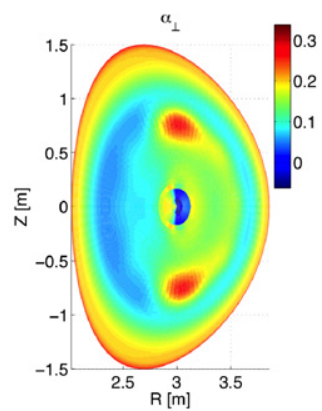

(c) $\mathrm{OA}$

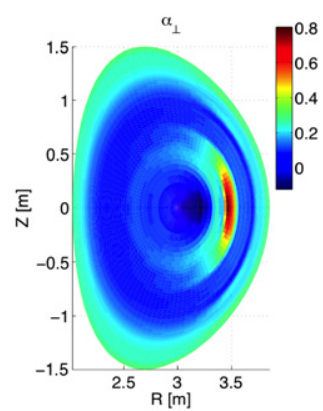

(d) LFS

Figure 12. Total $\alpha_{\perp}$ as defined in equation (7) initially and for the three converged solutions. Note the differences in scale.

Therefore, the passing particle current density from the model differs from the actual current density from VENUS, and it is also the main reason why the current is directly fed to the equilibrium from VENUS.

\subsection{Effects on the equilibrium}

For analysing the changes in the equilibrium, we will first define the minor radius $r$, the Shafranov shift $\Delta(r)$ and the shaping coefficients $S_{m}(r)$ using the expansion of the major radius:

$$
R=R_{0}+r \cos \theta-\Delta(r)+\sum_{m \geqslant 2} S_{m}(r) \cos (m-1) \theta .
$$

With this definition, the Shafranov shift vanishes at the magnetic axis, where $R=R_{0}$, and usually assumes a monotonically increasing profile. The Shafranov shift influences directly the form of a given equilibrium, whereas its second radial derivative is related to stability together with the parameter

$$
\alpha_{\perp} \equiv-\frac{2 R_{0} q^{2}}{B_{0}^{2}} \frac{\partial p_{\perp}}{\partial r},
$$

where $q$ denotes the safety factor. Indeed, $r \Delta^{\prime \prime}$ and $\alpha_{\perp}$ important quantities concerning ballooning modes [21-23] and the toroidal drift frequency of fast particles [23,24]. For the HFS case, the hot volume-averaged beta went from $3 \%$ to $30 \%$ of the thermal beta. For OA heating, the hot minority contribution went up to $60 \%$, and for the LFS heating the final hot reached $50 \%$ of the thermal beta. This means that the initially marginal hot particle beta has values comparable to the thermal beta at the end of the simulations, and can locally even dominate the thermal background. Even more important than the hot particle contribution to the total pressure are the steep perpendicular pressure gradients. Figure 12 shows $\alpha_{\perp}$ for the initial conditions and the three converged solutions. Note that the colour scaling is different for the different plots. It is clear that the changes are most important for LFS heating, where stability could indeed be affected.

The changes in pressure and current can have other effects on the equilibrium as well, such as the safety factor and the Shafranov shift. In particular, the total pressure influences the Shafranov shift, but pressure anisotropy also affects the plasma shaping [12], whereas the induced current can of course change the safety factor locally, which is the reason for using ICCD for MHD control. For these following considerations, one has to keep in mind that the 


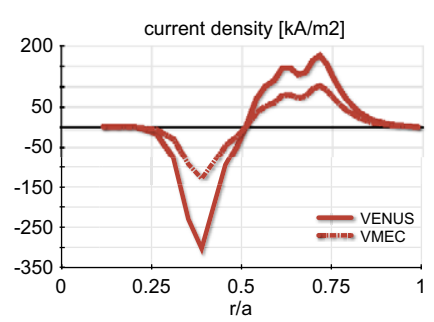

(a) HFS: Current



(b) OA: Current

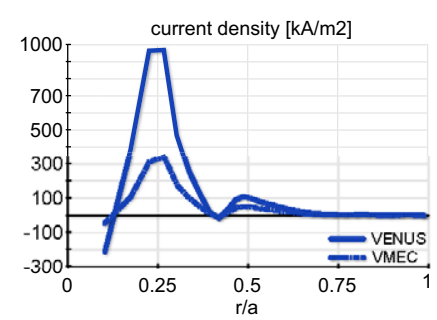

(c) LFS: Current

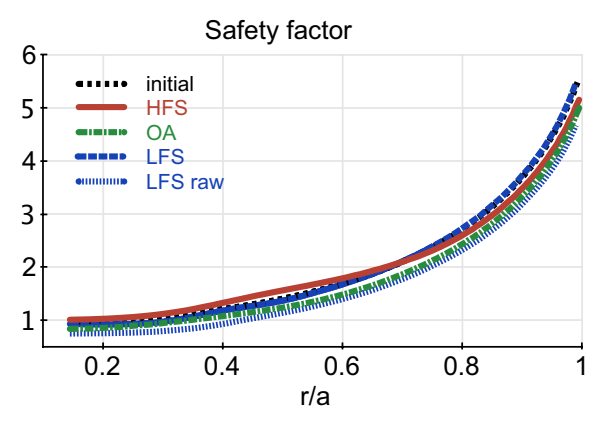

(d) Safety factor

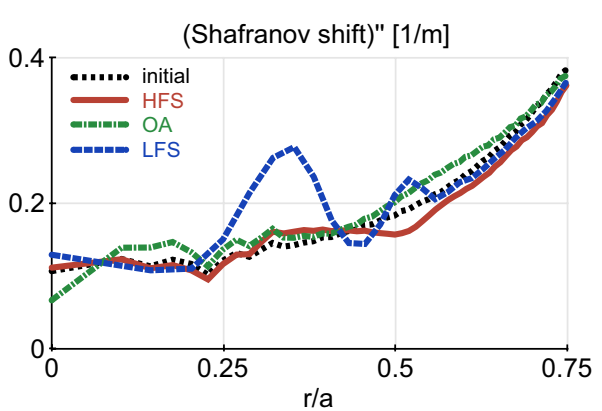

(e) $\Delta^{\prime \prime}(r)$

Figure 13. Direct RF and corrected current densities together with the resulting safety factor and $\Delta^{\prime \prime}$ profiles. The higher VENUS current densities from the OA and LFS cases are more diminished by the drag current than in the HFS case. Even so, the safety factor profiles are altered and $\Delta^{\prime \prime}$ shows large local variations, proving the importance of including the equilibrium in self-consistent computations.

current computed by VENUS was diminished considerably by the drag current assuming no impurities, equation (5), and in a more realistic case, where $Z_{\mathrm{eff}} \neq 1=Z_{h}$, the changes in the equilibrium will be larger. Figures $13(a)-(c)$ show the current density from VENUS together with the corrected current density fed to the equilibrium, and one can see that especially the large passing currents of the OA and LFS cases are very much diminished by the $\sqrt{r / R_{0}}$ dependence of the drag coefficient. Nevertheless, we find that the initial Shafranov shift of $13 \mathrm{~cm}$ changed during the simulations by $2 \%$ for the HFS, $5 \%$ for the OA cases, reaching a non-negligible change of $9 \%$ for the LFS cases. In figure $13(d)$ we show the local changes in the safety factor for all cases, and we have added the safety factor using the 'raw' RF current density, without drag current, in order to show the maximum effect at $Z_{\text {eff }} \rightarrow \infty$. Even with the drag current, one can see that the $q$-profile does change in these simulations. Due to the drag current without impurities, the HFS case is more effective here in terms of altering the safety factor profile than the LFS case, even if the total energy content is considerably smaller. The effect on the second derivative of the Shafranov shift, $\Delta^{\prime \prime}$, is shown in figure 13(e). Here, the pressure building up has its full effect on the magnetic flux surfaces, and $\Delta^{\prime \prime}(r)$ shows a very localized variation for the LFS case, which is due to the localized pressure of figure 13(c). These figures clearly show that changes in the equilibrium and its stability arise due to ICRH, and it is therefore important to be able to include them in self-consistent calculations.

\subsection{Inclusion of the equilibrium calculations in the iterative scheme}

In SCENIC, the equilibrium calculations are fully included in the iterative scheme, and the equilibrium is updated from iteration to iteration. We now want to determine whether this 


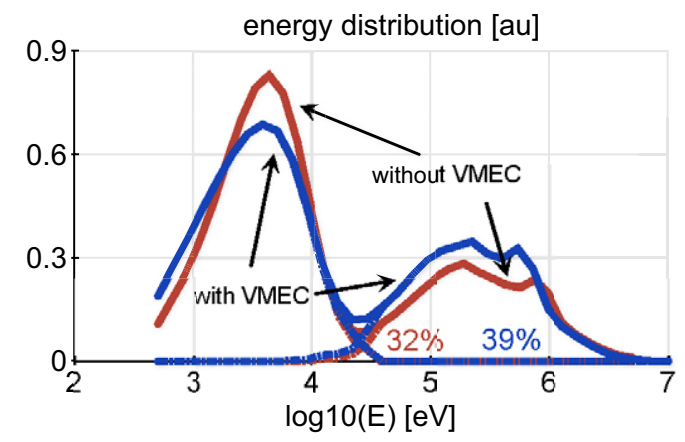

(a) Energy distributions $f(E) \sqrt{E}$. Without the equilibrium evolution in the iterative scheme, the final tails are lower, and more minority particles remain thermal.

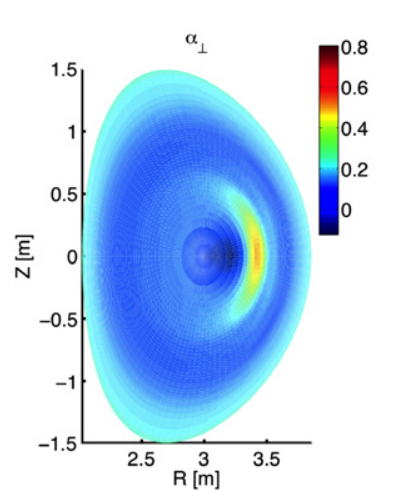

(b) The ballooning parameter $\alpha_{\perp}$, with the colors to scale with Fig. 12(d). The value of this parameter, which is important for global stability, is underestimated by a factor of two if the equilibrium is not updated during the simulation.


(c) The dielectric tensor element $\mathcal{E}_{n n}$ with the colors to scale with Fig. 10(c). The smaller tails have the effect that the dielectric tensor maxima are less localised than if the equilibrium is included in the iterative scheme. Moreover, the maxima are closer to the cold resonant layer than in Fig. 10(c)

Figure 14. Comparison of the final states for the LFS simulations with and without updating the equilibrium at the end of each iteration. For the simulation without updating, the equilibrium has been re-computed once the simulation including LEMan and VENUS converged. Clearly, the results are different, with a smaller high-energy tail for the case without inclusion of the equilibrium. As a result, equilibrium quantities such as $\alpha_{\perp}$ differ substantially.

inclusion is important or if it would be sufficient to base the wave and particle iterations, and their saturated solution, on an unchanged equilibrium. The final equilibrium could be constructed, including the fast ion effects in pressure and current, once a converged solution with unchanged equilibrium is obtained. We have repeated the simulations for the LFS case, keeping the equilibrium constant during the complete simulation, but still updating the wave fields with the thermal and hot minority contributions from VENUS. An immediate difference is in the number of iterations needed. Whereas the LFS simulation discussed in the previous sections required 22 iterations with a total duration of about 2.5 slowing down times, the simulation without the equilibrium evolution had to be iterated 30 times with a total duration of 3.5 slowing down times to achieve convergence. But most importantly, the high energy tails of the converged solution are lower, as can be observed in figure 14(a) Clearly, more minority 


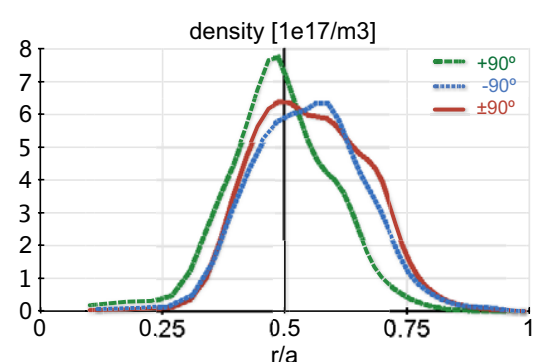

(a) Density

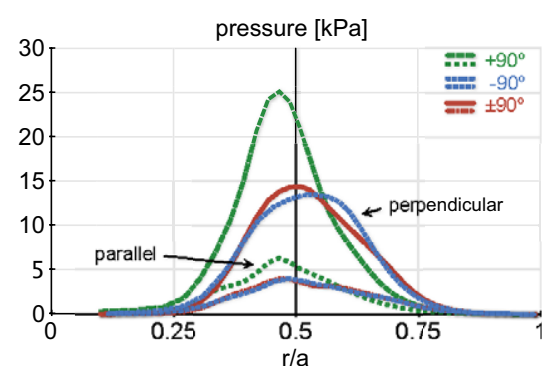

(b) Pressure

Figure 15. The RF-induced particle pinch causes density and pressure profiles to be peaked sharply inside (broadly outside) the resonant layer for co- (counter) current wave injection. Here, +17 corresponds to co-current injection. The solid vertical line marks the resonant layer, the upper curves on the right plot perpendicular, the lower curves parallel pressure.

particles remain thermal and do not contribute to the anisotropic high-energy tail. As a result, the effects on the equilibrium are substantially underestimated, as shown in figure $14(b)$. We show as an example the values of the ballooning parameter $\alpha_{\perp}$, which is a factor of two lower if one omits the equilibrium calculations in the iterative scheme during the simulations. A similar behaviour is visible on all equilibrium relevant quantities, showing the same general features, but the effects due to energetic particles are smaller if we omit the equilibrium evolution in the simulations. As a last example we plot the dielectric tensor element $\mathcal{E}_{\mathrm{nn}}$, where the colours in figure $14(c)$ are to scale with figure $10(c)$, allowing for direct comparison. Clearly, the maxima of the dielectric tensor are less localized in poloidal angle if we omit the equilibrium calculations, due to lower pressure anisotropy. To conclude this study, we can state that the equilibrium has to be included in self-consistent computations of ICRH, and has to be part of the self-consistent scheme, as variations of the equilibrium during the simulation alter, e.g., the particle orbits, as discussed in [24].

\section{RF-induced particle pinch}

For this last section we have run the HFS case not only using two waves with $n_{\phi}= \pm 17$, but also one simulation per wave, i.e. one with $n_{\phi}=+17$ and one with $n_{\phi}=-17$. We use the sign convention that a plus means co-current, a minus counter-current. The motivation is to check the RF-induced particle pinch, which has been observed and described, e.g., in [9, 25, 26]. Due to wave-particle interactions, one can show that we expect a net inward drift of resonant particles for co-current injection and a net outward drift for counter-current. As a result, co-current injection produces narrower but larger peaks inside the tangent resonant layer of density and pressure, whereas counter-current injection produces wider peaks outside the tangent resonant layer [10]. This is confirmed in figure 15, where the density (left plot) and most of all the perpendicular pressure (upper curves in the right plot) show a sharp peak inside the resonant layer (solid vertical line) for the co-current case, whereas the profile is much broader and peaked outside the resonant layer for counter-current injection.

\section{Conclusions}

In this work, we have used the code package SCENIC for self-consistent ion cyclotron resonant heating scenarios. Other than in similar numerical models, we have the unique 
possibility to include anisotropic equilibrium evolution within the model, and iterate the complete package of equilibrium, wave and full orbit following codes a sufficient number of times until convergence is achieved. With this, we investigated JET-like equilibria and hydrogen minority heating schemes with $3 \%$ hydrogen in background deuterium. $12 \mathrm{MW} \mathrm{RF}$ power scenarios with symmetric wave spectra were studied and high-energy tails in the MeV range were obtained. Then, we investigated the effects of such a highly energetic anisotropic species on the distribution function, the dielectric tensor and the equilibrium, finding that even without considering impurities, the induced currents can alter the safety factor profile, and the arising pressure changes the Shafranov shift and its derivatives, changing the stability characteristics in turn. The model includes a vast number of physical mechanisms, and as an example the RF-induced particle pinch could be elucidated. These studies show clearly that self-consistent modelling of ICRH should include the evolution of the distribution function, the dielectric tensor and the equilibrium.

\section{Acknowledgments}

This work was supported in part by the Swiss National Science Foundation. We thank Dr S P Hirshman for the use of the VMEC code. These results have been obtained using the high performance clusters HPC-FF at the Jülich Supercomputing Center (JSC) and Rosa XT-5 at the Swiss National Supercomputing Centre.

Euratom (C) 2011.

\section{Appendix. Anisotropic dielectric tensor}

The dielectric tensor implemented in the wave code LEMan for the hot minority species is based on the distribution function given in equation (1), and takes the explicit form [2]

- For $B \geqslant B_{\mathrm{c}}$ :

$$
\begin{aligned}
& \mathcal{E}_{\mathrm{nn}}=1-\frac{1}{2 \omega} \frac{\sqrt{T_{\|} / T_{\perp}}}{C_{+}} \sum_{k}\left(\tilde{Z}_{1}^{\|}+\tilde{Z}_{-1}^{\|}\right) \\
& \mathcal{E}_{\mathrm{nb}}=-\frac{\mathrm{i}}{2 \omega} \frac{\sqrt{T_{\|} / T_{\perp}}}{C_{+}} \sum_{k}\left(\tilde{Z}_{1}^{\|}-\tilde{Z}_{-1}^{\|}\right)=-\mathcal{E}_{\mathrm{bn}} \\
& \mathcal{E}_{\|\|}=1+\frac{2}{\left(k_{\|} v_{\| T}\right)^{2}} \frac{\sqrt{T_{\|} / T_{\perp}}}{C_{+}} \sum_{k}\left(\tilde{\omega}_{\mathrm{p}}^{2}-\omega \tilde{Z}_{0}^{\|}\right),
\end{aligned}
$$

- For $B<B_{\mathrm{c}}$ :

$$
\begin{aligned}
& \mathcal{E}_{\mathrm{nn}}=\mathcal{E}_{\mathrm{nn}}^{B \geqslant B_{\mathrm{c}}}-\frac{1}{2 \omega} \frac{C_{+}-C_{-}}{C_{+} C_{-}} \sum_{k}\left(\tilde{Z}_{1}^{\perp}+\tilde{Z}_{-1}^{\perp}\right) \\
& \mathcal{E}_{\mathrm{nb}}=\mathcal{E}_{\mathrm{nb}}^{B \geqslant B_{\mathrm{c}}}-\frac{\mathrm{i}}{2 \omega} \frac{C_{+}-C_{-}}{C_{+} C_{-}} \sum_{k}\left(\tilde{Z}_{1}^{\perp}-\tilde{Z}_{-1}^{\perp}\right)=-\mathcal{E}_{\mathrm{bn}} \\
& \mathcal{E}_{\|\|}=\mathcal{E}_{\|\|}^{B \geqslant B_{\mathrm{c}}}-\frac{C_{+}+C_{-}}{C_{+} C_{-}} \sum_{k}\left(\sqrt{\frac{B_{\mathrm{c}}-B}{B_{\mathrm{c}}}} \tilde{\omega}_{\mathrm{p}}^{2}-\omega \tilde{Z}_{0}^{\perp}\right) .
\end{aligned}
$$


Here, $v_{\| T}^{2}=2 T_{\|} / m$ is the fast particle thermal parallel velocity and

$$
\begin{aligned}
& C_{ \pm}=\frac{B_{\mathrm{c}}}{B} \pm \frac{T_{\perp}}{T_{\|}}\left(1-\frac{B_{\mathrm{c}}}{B}\right) \\
& Z^{\mathrm{Sh}}(z)=\frac{z}{\sqrt{\pi}} \int_{-\infty}^{\infty} \frac{1}{z-x} \mathrm{e}^{-x^{2}} \mathrm{~d} x, \quad \operatorname{Im} z>0 \\
& \tilde{Z}_{l}^{\|}=\frac{\tilde{\omega}_{\mathrm{p}}^{2}}{\omega-l \Omega_{\mathrm{c}}} Z^{\mathrm{Sh}}\left(\frac{\omega-l \Omega_{\mathrm{c}}}{k_{\|} v_{\| T}}\right) \\
& \tilde{Z}_{l}^{\perp}=\sqrt{\frac{B_{\mathrm{c}}-B}{B_{\mathrm{c}}}} \frac{\tilde{\omega}_{\mathrm{p}}^{2}}{\omega-l \Omega_{\mathrm{c}}} Z^{\mathrm{Sh}}\left(\sqrt{\frac{B_{\mathrm{c}}}{B_{\mathrm{c}}-B}} \frac{\omega-l \Omega_{\mathrm{c}}}{k_{\|} v_{\| T}}\right),
\end{aligned}
$$

and $\tilde{\omega}_{\mathrm{p}}^{2}=q_{k}^{2} \mathcal{N}_{k} / \epsilon_{0} m_{k}$ the plasma frequency of species $k$. The subscripts $n, b$ and $\|$ denote the normal, bi-normal and parallel components relative to the magnetic field.

\section{References}

[1] Graves J P et al 2010 Nucl. Fusion 50052002

[2] Jucker M et al 2010 Integrated modeling for ion cyclotron resonant heating in toroidal systems Comput. Phys. Commun. at press

[3] Cooper W A et al 2006 Nucl. Fusion 46683

[4] Popovich P, Cooper W A and Villard L 2006 Comput. Phys. Commun. 175250

[5] Fischer O, Cooper W A, Isaev M Y and Villard L 2002 Nucl. Fusion 42817

[6] Hedin J, Hellsten T, Eriksson L-G and Johnson T 2002 Nucl. Fusion 42527

[7] Jaeger E F et al 2006 Nucl. Fusion 46 S397

[8] Choi M et al 2010 Phys. Plasmas 17056102

[9] Chen L, Vaclavik J and Hammett G W 1988 Nucl. Fusion 28389

[10] Eriksson L-G and Schneider M 2005 Phys. Plasmas 12072524

[11] Devoto R S et al 1992 Nucl. Fusion 32773

[12] Madden N A and Hastie R J 1994 Nucl. Fusion 34519

[13] Fisch N J 1987 Rev. Mod. Phys. 59175

[14] Graves J P et al 2010 Phys. Plasmas 17056118

[15] Cooper W A et al 2009 Comput. Phys. Commun. 1801524

[16] Hellsten T, Carlsson J and Eriksson L-G 1995 Phys. Rev. Lett. 743612

[17] Carlsson J, Hellsten T and Hedin J 1998 Phys. Plasmas 52885

[18] Laxåback M and Hellsten T 2005 Nucl. Fusion 451510

[19] Connor J W and Cordey J G 1974 Nucl. Fusion 14185

[20] Graves J P et al 2009 Phys. Rev. Lett. 102065005

[21] Bishop C M and Hastie R J 1985 Nucl. Fusion 251443

[22] Connor J W, Hastie R J and Taylor J B 1978 Phys. Rev. Lett. 40396

[23] Rosenbluth M N, Tsai S T, Dam J W V and Engquist M G 1983 Phys. Rev. Lett. 511967

[24] Jucker M, Graves J P, Cooper G A and Cooper W A 2008 Plasma Phys. Control. Fusion 50065009

[25] Eriksson L-G et al 1998 Phys. Rev. Lett. 811231

[26] Eriksson L-G et al 2004 Phys. Rev. Lett. 92235001 